\title{
Análise e projeção da salubridade ambiental com base em planos municipais de saneamento básico
}

\section{Analysis and projection of environmental health based on basic sanitation municipal plans}

Data de entrada: $12 / 08 / 2016$

- Data de aprovação: 08/05/2017

\section{Resumo}

Este trabalho apresenta uma metodologia simplificada para avaliação das condições de salubridade dos municípios por meio de um Índice de Salubridade Ambiental (ISA), que considera o nível de atendimento dos serviços de saneamento básico, conforme detalhado nos seus planos municipais de saneamento básico, englobando tanto as zonas urbanas como as zonas rurais. $O$ estudo fez uma avaliação dessa metodologia em dez municípios do Estado do Ceará, com populações variando entre 7 e 265 mil habitantes. Os resultados mostraram que as zonas rurais apresentam consistentemente níveis de salubridade inferiores aos das zonas urbanas, sendo $10 \%$ dos municípios classificados como insalubres, $50 \%$ de baixa salubridade e $40 \%$ de média salubridade. Verificou-se que quanto maior o município, melhor a salubridade ambiental. Observou-se também que a existência de Sistema Integrado de Saneamento Rural (SISAR) foi um fator relevante para a classificação da salubridade ambiental dos municípios de menor porte. Outro resultado impactante foi que somente a partir de um horizonte de planejamento de médio prazo (20 anos) os municípios poderão atingir a condição de salubres. Combinando-se os dados do presente estudo com trabalhos anteriores, foi possível obter uma correlação geral capaz de descrever o ISA em função da população dos municípios. Extrapolando essa correlação para os 184 municípios do Estado do Ceará, pode-se inferir que a grande maioria dos municípios (84\%) foi classificada como de baixa salubridade. Finalmente, a metodologia proposta pode servir como uma ferramenta para priorização de programas de saneamento em função do ISA, conforme preconizado no Plano Nacional de Saneamento Básico (PNSB). Palavras-chave: Salubridade ambiental. Saneamento básico. Planejamento.

\section{Abstract}

This paper presents a simplified methodology for assessing the environmental health conditions of the municipalities through an Environmental Health Index, which considers the level of attendance of basic sanitation services, as detailed in its municipal basic sanitation plans, encompassing both the urban and rural areas. The study made an assessment of this methodology in ten municipalities of the state of Ceara, Brazil, with populations ranging from 7,000 to 265,000 inhabitants. The results showed that rural areas consistently show health levels lower than those of urban areas, $10 \%$ of municipalities classified as unhealthy, $50 \%$ of low health and $40 \%$ of average health. It was found that

\footnotetext{
Jaime Silva Mendes* - Engenheiro Civil (Universidade Federal do Ceará).

Iran E. Lima Neto - Engenheiro Civil (Universidade Federal do Ceará), mestre em Hidráulica e Saneamento (Escola de Engenharia de São Carlos/Universidade de São Paulo), PhD em Engenharia Hidráulica (Universidade de Alberta/Canadá) e professor Adjunto (Departamento de Engenharia Hidráulica e Ambiental/UFC).

*Endereço para correspondência: Departamento de Engenharia Hidráulica e Ambiental/UFC - Campus do Pici - Bloco $713,1^{\circ}$ andar - CEP 60451-970 - Fortaleza (CE).E-mail: iranadeha.ufc.br
} 
the larger the municipality, the better the environmental health. It was also observed that the existence of Integrated Rural Sanitation Systems (SISAR) was a relevant factor for the environmental health classification of smaller municipalities. Another striking result was that only from a medium-term planning horizon (20 years) the municipalities can achieve healthy condition. Combining the data from this study with previous studies, it was possible to obtain a general correlation to describe the Environmental Health Index as a function of the population of the municipalities. Extrapolating this correlation to the 184 municipalities in the state of Ceara, one can infer that the vast majority of municipalities (84\%) was classified as low health. Finally, the proposed methodology can serve as a tool for prioritizing sanitation programs according to the Environmental Health Index, as recommended by the National Sanitation Plan (PNSB). Keywords: Environmental health. Basic sanitation. Planning.

\section{INTRODUÇÃO}

O saneamento básico é definido como o conjunto de serviços de abastecimento de água potável, esgotamento sanitário, drenagem e manejo das águas pluviais urbanas e limpeza urbana e manejo de resíduos sólidos, que visam promover a saúde humana e o desenvolvimento sustentável. Portanto, para verificar as condições de vida de uma população, é fundamental que sejam levantadas as questões relacionadas à infraestrutura de saneamento básico instalada e à qualidade da prestação desses serviços (PHILIPPI JR., 2005).

Diversos municípios brasileiros, principalmente do Nordeste, sofrem com a falta de água potável para abastecimento humano, mais acentuada nas zonas rurais, sem falar que os índices são ainda piores em relação aos demais setores do saneamento básico. Sem dúvida o ponto de partida para tentar solucionar os problemas do setor de saneamento básico é um bom planejamento das ações e medidas a serem tomadas. Com a Lei Federal $11.445 / 2007$, que estabelece diretrizes nacionais para o saneamento básico, e o Decreto Federal $n^{\circ} 7.217 / 2010$, que a regulamenta, houve novas perspectivas para um planejamento de qualidade no setor em nível municipal, regional e federal. A Lei 11.445/2007 determina em seu art. 9 a elaboração dos planos de saneamento básico por parte do titular dos serviços como condição básica para o acesso a recursos financeiros da União. Exemplos de trabalhos anteriores sobre o desen- volvimento de planos de saneamento básico nos termos da legislação supracitada foram apresentados por Lima Neto (2011), Lima Neto e Santos (2011), Lima Neto (2013), Galvão Júnior (2014), e Pereira e Heller (2015).

No setor de saneamento básico, os indicadores e índices são fundamentais para visualizar a qualidade dos serviços prestados, além de se prestarem à avaliação das relações entre saneamento e outras áreas. Várias correlações são realizadas entre os indicadores de saneamento, saúde, meio ambiente, área socioeconômica, entre outras, visando encontrar os impactos que um setor ocasiona em outro (HELLER, 1997; TEIXEIRA et al., 2011). Entre estes, destacam-se os indicadores utilizados para avaliar a salubridade ambiental, que é definida como "a qualidade ambiental capaz de prevenir a ocorrência de doenças veiculadas pelo meio ambiente e de promover o aperfeiçoamento das condições mesológicas favoráveis à saúde da população urbana e rural" (PHILIPPI JR. et al., 2005). Portanto, conforme disposto no Decreto $n^{\circ} 7.217 / 2010$, é de fundamental importância o desenvolvimento de estudos e a consolidação de metodologia que possibilitem caracterizar e avaliar a situação de salubridade ambiental no território nacional, por bacias hidrográficas e por municípios. Cabe salientar que o Plano Nacional de Saneamento Básico (PNSB) enfatiza que um dos critérios para priorização dos programas relacionados ao setor é selecionar municípios com indicadores críticos de salubridade ambiental. Diversos autores 
aplicaram ou desenvolveram um Índice de Salubridade Ambiental (ISA), sendo cada metodologia voltada para uma área específica, como: áreas urbanas, áreas rurais, ocupações espontâneas, favelas, entre outras (CONESAN, 1999; DIAS et al., 2004; BATISTA e SILVA, 2006; RIBEIRO, 2007; COSTA, 2010; BUCKLEY e DALTRO FILHO, 2012; CUNHA, 2012; VALVASSORI e ALEXANDRE, 2012). Entretanto, a literatura ainda carece de um ISA geral que englobe toda a população urbana e rural dos municípios, como ferramenta para hierarquização e priorização de programas de saneamento em função desse índice, como preconizado no PNSB.

Nesse contexto, apresenta-se neste trabalho uma metodologia simplificada para calcular um Índice de Salubridade Ambiental, considerando tanto as zonas urbanas como as zonas rurais dos municípios. Trata-se de uma adaptação do ISA proposto inicialmente pelo Conselho de Saneamento do Estado de São Paulo (CONESAN, 1999), que permite avaliar a salubridade ambiental de municípios em função apenas de índices de saneamento básico, os quais podem ser facilmente obtidos a partir de Planos de Saneamento Básico existentes. A metodologia proposta será aplicada a dez municípios do Estado do Ceará. Será avaliada não apenas a salubridade ambiental atual dos municípios, mas também a sua projeção ao longo de 30 anos. Além disso, serão obtidas correlações que permitirão a extrapolação dos resultados para uma avaliação geral da salubridade ambiental do Ceará, ou ainda para hierarquização dos municípios, conforme previsto no PNSB.

\section{METODOLOGIA}

A área de estudo compreendeu os municípios de Altaneira, Barbalha, Caririaçu, Crato, Farias Brito, Jardim, Juazeiro do Norte, Missão Velha, Nova Olinda e Santana do Cariri, situados nas bacias hidrográficas do Alto Jaguaribe e Salgado, na porção centro-sul do Estado do Ceará. A Figura 1 mostra a região de estudo delimitada.

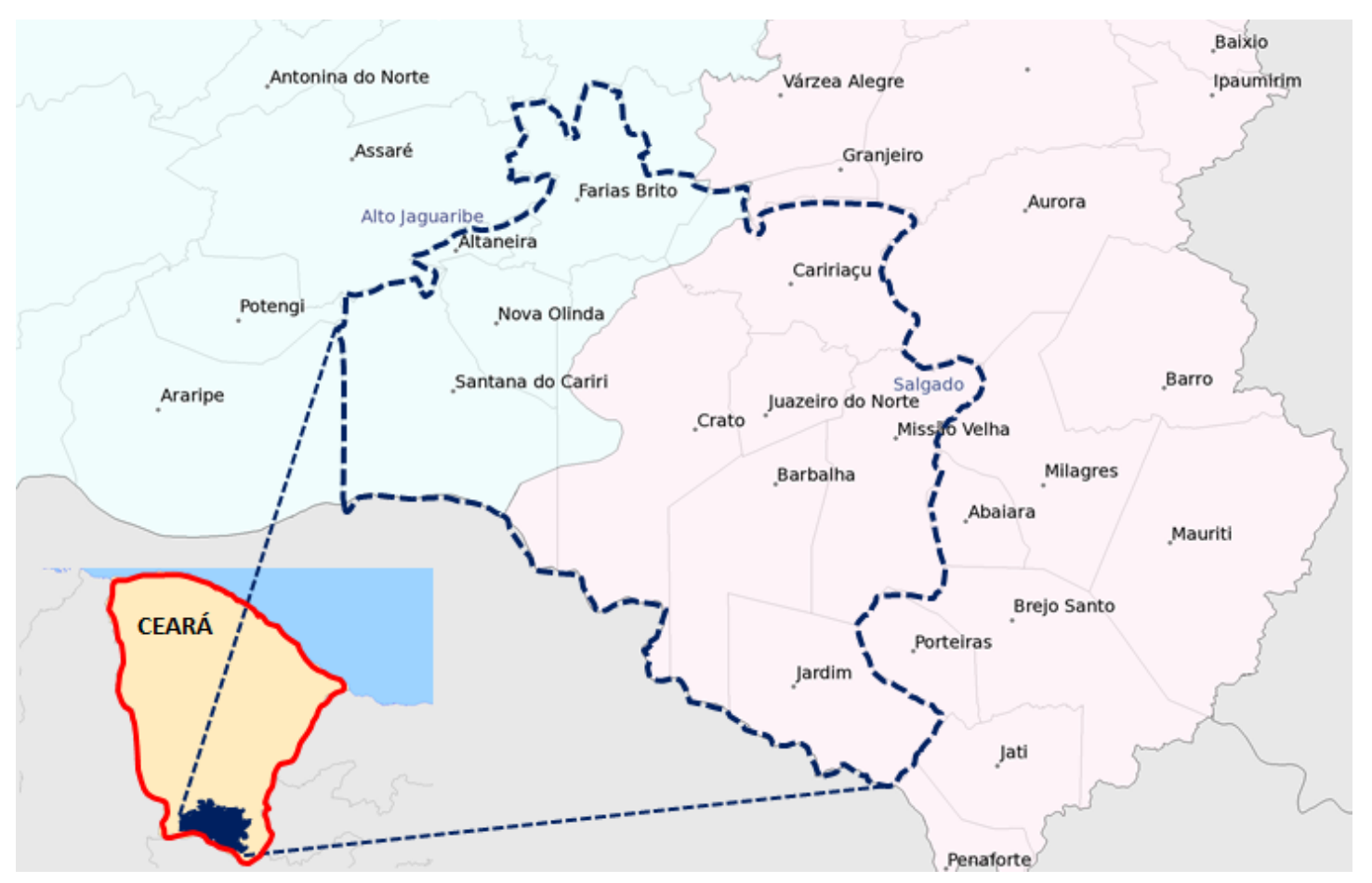

Figura 1 - Mapa destacando os dez municípios selecionados, os quais estão situados nas bacias hidrográficas do Alto Jaguaribe e Salgado, na porção centro-sul do Estado do Ceará. (Adaptado de SRH-CE, 2016) 
Os dados utilizados nesta pesquisa foram obtidos dos Planos Municipais de Saneamento Básico - PMSB dos dez municípios supracitados, os quais foram finalizados e aprovados em 2012. Esses municípios possuíam no referido ano populações totais variando entre aproximadamente 7 mil e 265 mil habitantes, conforme detalhado na Tabela 1.

Tabela 1 - Dados populacionais dos municípios selecionados

\begin{tabular}{|c|c|}
\hline Município & População (hab) \\
\hline Altaneira & 7.081 \\
\hline Barbalha & 57.544 \\
\hline Caririaçu & 27.757 \\
\hline Crato & 125.241 \\
\hline Farias Brito & 19.612 \\
\hline Jardim & 27.042 \\
\hline Juazeiro do Norte & 265.415 \\
\hline Missão Velha & 35.001 \\
\hline Nova Olinda & 14.725 \\
\hline Santana do Cariri & 17.359 \\
\hline
\end{tabular}

No presente trabalho, procurou-se relacionar diretamente a salubridade ambiental com os indicadores de saneamento básico, que é o escopo dos PMSB. A seguir, apresenta-se uma descrição da metodologia proposta.

\subsection{Indice de Salubridade Ambiental para Zonas Urbanas - ISA/Urbano}

Em geral, os Planos Municipais de Saneamento Básico elaborados nos termos da Lei Federal 11.445/2007 e do Decreto Federal n 7.217/2010 dispõem apenas de indicadores relacionados ao próprio setor de saneamento básico: abastecimento de água, esgotamento sanitário, limpeza urbana e manejo de resíduos sólidos, e drenagem e manejo das águas pluviais urbanas. Nesse sentido, formulou-se uma expressão para o ISA/ Urbano como a média ponderada desses quatro subindicadores, como se apresenta na Equação 1:

$$
\text { ISA/Urbano }=0,35 \mathrm{I}_{\mathrm{ab}}+0,25 \mathrm{I}_{\mathrm{es}}+0,25 \mathrm{I}_{\mathrm{rs}}+0,15 \mathrm{I}_{\mathrm{du}}
$$

Sendo:

$\mathrm{I}_{\mathrm{ab}}=$ Indicador de abastecimento de água (\% de domicílios atendidos por sistema de abastecimento de água potável, incluindo rede e poços);

$\mathrm{I}_{\text {es }}=$ Indicador de esgotamento sanitário (\% de domicílios atendidos por sistema de esgotamento sanitário, incluindo rede e fossas sépticas);

$\mathrm{I}_{\mathrm{rs}}=$ Indicador de resíduos sólidos (\% de domicílios atendidos por serviço de coleta e manejo dos resíduos sólidos);

$\mathrm{I}_{\mathrm{du}}=$ Indicador de drenagem urbana (\% de domicílios atendidos por serviço de drenagem e manejo de águas pluviais urbanas).

Os pesos relativos na Equação (1) foram dados de acordo com a relevância que cada setor representa potencialmente para a vida população e a qualidade do ambiente. $\mathrm{A}$ água teve peso maior por ser um elemento essencial à vida. Os setores de esgoto e resíduos sólidos tiveram pesos idênticos por se considerar que impactam de forma semelhante a vida da população. Já a drenagem teve menor peso por seu impacto somente ser relevante em eventos de chuvas intensas, que ocorrem com baixa frequência em regiões semiáridas, como o Estado do Ceará. Vale destacar que os pesos supracitados são semelhantes àqueles sugeridos pelo Conselho de Saneamento do Estado de São Paulo (CONESAN, 1999) e, posteriormente, por Batista e Silva (2006). No entanto, os pesos referentes aos subindicadores relacionados aos aspectos socioeconômicos, de controle de vetores e de recursos hídricos (ver Batista e Silva, 2006), os quais normalmente não são disponibilizados nos PMSB, foram desprezados do ISA (Equação 1). Essa simplificação justifica-se pelo fato de tais aspectos já estarem correlacionados aos quatros setores do saneamento básico. 
Para indicar a situação de salubridade ambiental segundo os resultados obtidos com a Equação 1, foram utilizadas as faixas de pontuação da Tabela 2:

Tabela 2 - Nível de salubridade ambiental por faixa de situação.

\begin{tabular}{|c|c|}
\hline Nível de Salubridade & Pontuação do ISA \\
\hline Insalubre & $0-25$ \\
\hline Baixa salubridade & $25-50$ \\
\hline Média salubridade & $50-75$ \\
\hline Salubridade aceitável & $75-90$ \\
\hline
\end{tabular}

Os valores apresentados na Tabela 2 foram baseados em Batista e Silva (2006), e são inversamente proporcionais às taxas de ocorrência de doenças relacionadas à carência do saneamento básico. A adaptação em relação ao modelo de Batista e Silva (2006) foi o desmembramento da última categoria em duas classes: salubridade aceitável e salubre. Essa adaptação foi necessária, uma vez que foi observada uma tendência geral de redução das taxas supracitadas em áreas com ISA superior a 90. Logo, pode-se dizer que a condição "Salubre" representa as áreas com menor incidência dessas doenças e, portanto, com melhor qualidade de vida da população quanto à salubridade ambiental.

\section{2 Índice de Salubridade Ambiental para Zonas Rurais - ISA/Rural}

O ISA/Rural também teve sua formulação englobando subindicadores do saneamento básico, conforme mostrado na Equação 2:

$\mathrm{ISA} /$ Rural $=0,40 \mathrm{I}_{\mathrm{ab}}+0,30 \mathrm{I}_{\mathrm{es}}+0,30 \mathrm{I}_{\mathrm{rs}}$
Os pesos de 0,40 para o setor de água e 0,30 para cada um dos outros dois setores, esgoto e resíduos sólidos, foram novamente fundamentos na hipótese de a primeira ser de maior relevância para as condições de salubridade ambiental, pois a água é elemento indispensável à existência humana. Os outros dois setores tiveram seus pesos iguais por potencialmente impactarem a vida da população e a qualidade do ambiente de forma semelhante, conforme proposto para as zonas urbanas. Ressalta-se que no ISA/Rural não existe subindicador de drenagem urbana, uma vez que as zonas rurais apresentam em geral baixa impermeabilização do solo e baixa densidade demográfica. Logo, pode-se considerar que a drenagem de águas pluviais não é um problema relevante nas zonas rurais. De fato, os efeitos da poluição difusa causados por cheias/inundações/alagamentos somente são significativos nas zonas urbanas dos municípios do Ceará.

Assim, a classificação das zonas rurais quanto à salubridade ambiental também pode ser obtida com base nas faixas de pontuação da Tabela 2 .

\section{3 İndice de Salubridade Ambiental Municipal \\ - ISA/Município}

Para obter um Índice de Salubridade Ambiental geral para o município, pode-se utilizar a Equação 3 , que consiste em uma média ponderada pela população dos ISA/Urbano e ISA/Rural:

ISA/Município $=\left[\left(\right.\right.$ Pop $_{\text {urbana }} \times I S A /$ Urbano $)+\left(\right.$ Pop $_{\text {rural }}$ $\mathrm{x}$ ISA/Rural)]/Pop total

Sendo:

Pop $_{\text {urbana }}=$ população urbana do município; 
Pop $_{\text {rural }}=$ população rural do município;

Pop $_{\text {total }}=$ população total do município.

Por fim, a qualidade da salubridade ambiental do município pode ser classificada de acordo com a Tabela 2.

No presente trabalho, tomando como base os seus Planos de Saneamento Básico, pode-se avaliar e comparar o ISA/Urbano, ISA/Rural e ISA/ Município dos dez municípios do Estado do Ceará supracitados. Além disso, de posse das projeções dos índices de atendimento de cada setor do saneamento básico, foi possível prever o comportamento do ISA ao longo de diferentes horizontes de planejamento (até 30 anos).

Adicionalmente, os dados desta pesquisa foram combinados com os dados de trabalhos anteriores para, por meio de análise de regressão, gerar uma correlação geral que fosse capaz de descrever o ISA em função da população dos municípios. Essa correlação foi aplicada aos 184 municípios do Estado do Ceará para avaliar os seus níveis de salubridade.

\section{RESULTADOS E DISCUSSÃO}

Após pesquisa nos Planos Municipais de Saneamento Básico - PMSB de Altaneira, Barbalha, Caririaçu, Crato, Farias Brito, Jardim, Juazeiro do Norte, Missão Velha, Nova Olinda e Santana do Cariri/CE, pode-se aplicar as Equações (1), (2) e (3) para avaliar o Índice de Salubridade Ambiental para as zonas urbanas (ISA/Urbano), rurais (ISA/Rural) e para os referidos municípios como um todo (ISA/Município). A Figura 2 mostra os resultados obtidos. Percebe-se que as zonas ru- rais apresentam consistentemente níveis de salubridade significativamente inferiores aos das zonas urbanas, sendo a maior disparidade observada para o município de Jardim, cujo ISA/Urbano $=85,4$, enquanto o ISA/Rural $=2,2$. Pode-se inferir que isso é reflexo de uma política de concentração de investimentos em saneamento básico nas zonas urbanas, conforme reportado por Vargas (2011) para os municípios brasileiros em geral. Logo, como em muitos casos os esgotos e resíduos sólidos são dispostos de forma inadequada no meio rural, a salubridade ambiental fica dependendo basicamente do nível do serviço de abastecimento de água. Assim, municípios como Jardim, que tem um baixo índice de atendimento com rede de distribuição de água tratada (<5\%), possuirão um ISA/Rural inferior aos de municípios como Altaneira, que possui um índice de atendimento com rede significativamente superior (> $85 \%$ ). Vale destacar que na zona rural dos municípios estudados, assim como na maioria dos municípios do Ceará, o Sistema Integrado de Saneamento Rural - SISAR, que é um órgão não governamental, sem fins lucrativos, formado pela associação das comunidades, é em geral o responsável pela prestação do serviço de abastecimento de água. Por outro lado, nas zonas urbanas, os serviços de abastecimento de água e esgotamento sanitário são geralmente prestados pela companhia estadual (Cagece) ou por autarquias municipais (SAAE's), enquanto os serviços de limpeza urbana e manejo de resíduos sólidos e drenagem e manejo das águas pluviais urbanas são prestados pelas prefeituras municipais. Não foram observadas tendências claras de variação do ISA em função do tipo de prestador dos serviços. 


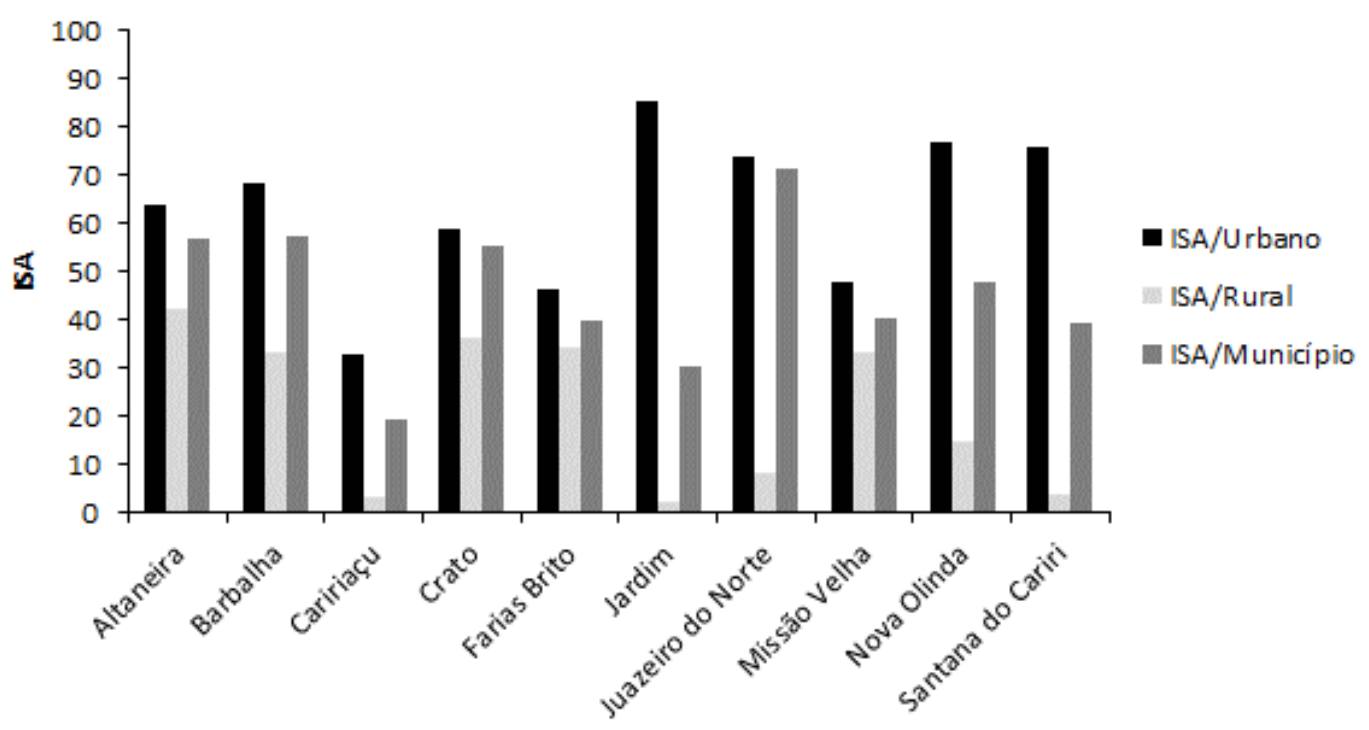

Figura 2 - Índices de Salubridade Ambiental para os dez municípios selecionados.

A Figura 3 mostra uma síntese dos resultados apresentados na Figura 2. Para as zonas urbanas (Fig. 3a), verifica-se que $40 \%$ dos municípios selecionados foram classificados como de média salubridade, enquanto os demais foram igualmente classificados como de baixa salubridade (30\%) e de salubridade aceitável (30\%). Esses resultados são piores que os relatados por Batista e Silva (2006) para a zona urbana do município de João Pessoa/PB e que os relatados por Valvassori e Alexandre (2012) para a zona urbana do município de Criciúma/SC, cujas áreas foram classificadas como de média salubridade a salubres. Em contraste, nas zonas rurais (Fig. 3b), metade dos municípios foi classificada como insalubre e a outra metade como de baixa salubridade. Salienta-se que esses resultados foram semelhantes aos relatados por Dias et al. (2004), Ribeiro (2007), Costa (2010), Cunha (2012), e Buckley e Daltro Filho (2012) para zonas rurais, de baixa renda ou favelas. Finalmente, a Fig. 3c mostra que os municípios foram em geral (ISA/Município) classificados como de média salubridade (50\%), baixa salubridade ( $40 \%)$ e insalubre (10\%). Isso revela que pelo menos a metade dos municípios estudados se encontra em uma condição crítica de salubridade ambiental. 
(a)

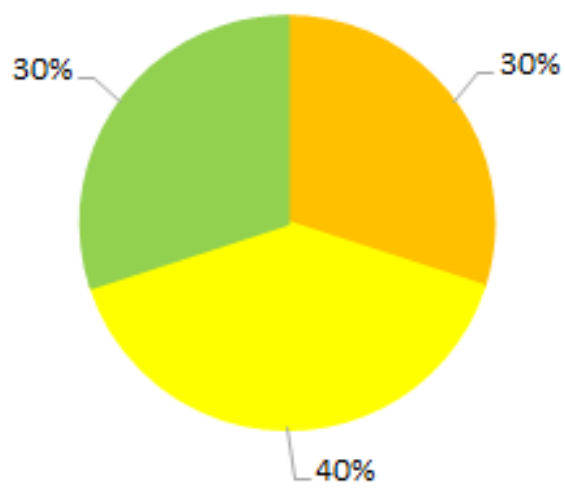

Baixa salubridade Média salubridade Salubridade ace itável

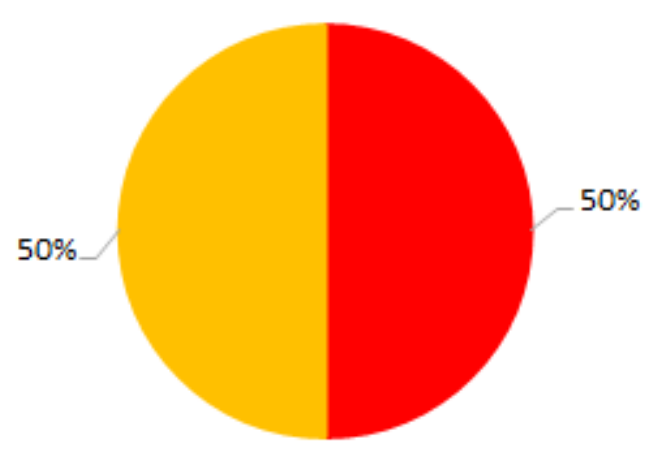

(b)

Insalubre Baixa salubridade

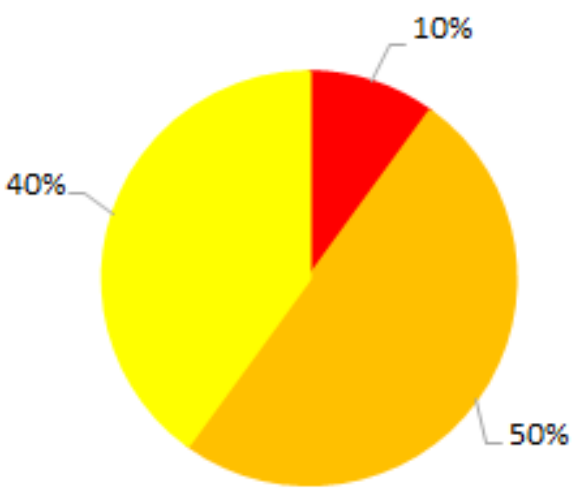

(c)

Insalubre Baixa salubridade Média salubridade

Figura 3 - Síntese da salubridade ambiental dos dez municípios selecionados:

(a) ISA/Urbano, (b) ISA/Rural, e (c) ISA/Município. 
A Figura 4 mostra uma projeção do ISA/Município ao longo dos horizontes de planejamento (metas emergenciais, de curto, médio e longo prazo) adotados nos Planos de Saneamento Básico dos dez municípios estudados. Nota-se que a partir de um horizonte de planejamento de médio prazo (20 anos), os municípios de Barbalha, Crato e Jua- zeiro do Norte (mais populosos) poderão atingir a condição de salubre, enquanto Altaneira, Caririaçu, Farias Brito e Missão Velha somente atingirão essa condição em um horizonte de longo prazo (30 anos). Já os demais municípios não atingirão a condição de salubre dentro dos prazos estabelecidos no PMSB.

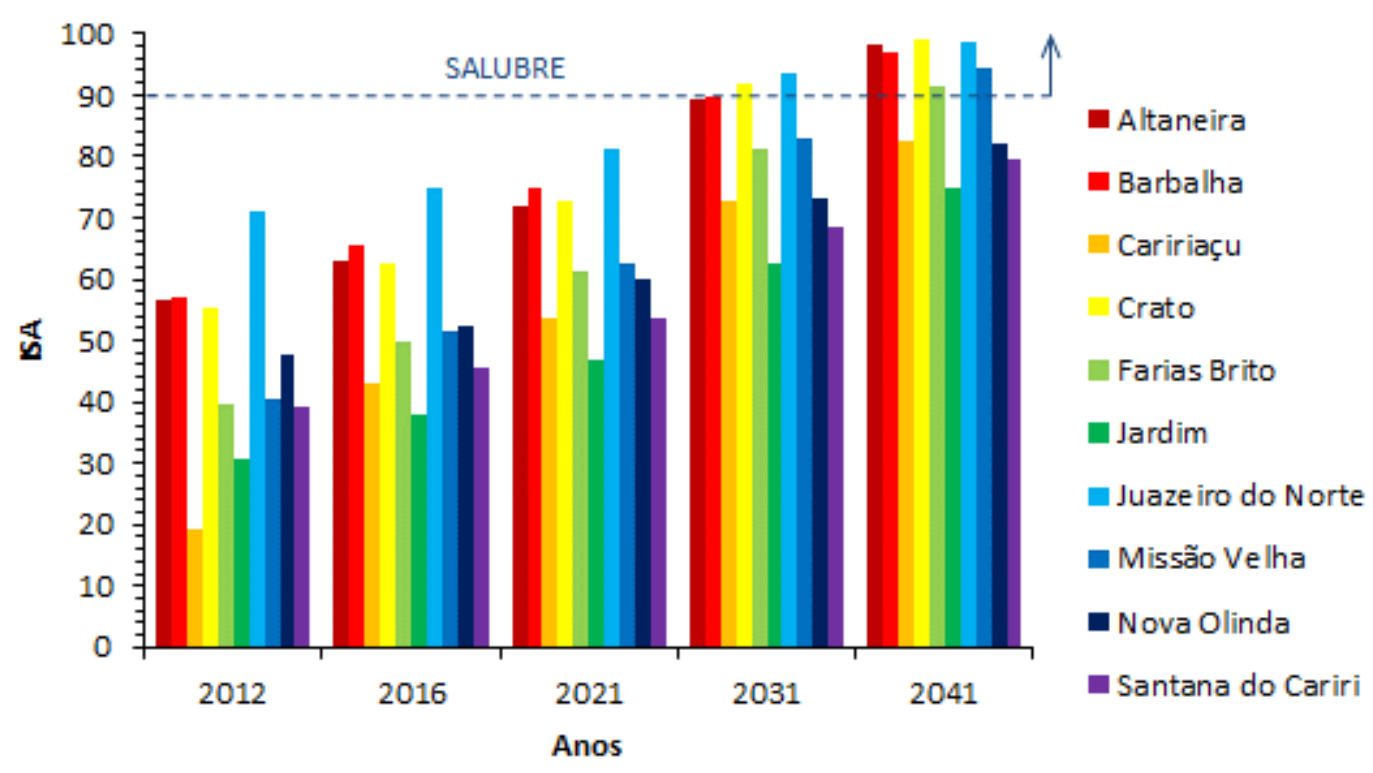

Figura 4 - Projeção do Índice de Salubridade Ambiental (ISA/Município) para os dez municípios selecionados.

Combinando os dados do presente estudo com aqueles obtidos no PMSB de outros municípios do Estado do Ceará (Araripe, Cariús, Fortaleza, Iguatu, Limoeiro do Norte e Morada Nova), pode-se obter por meio de análise de regressão (R2 = 0,84) uma correlação geral dada pela Equação (4) que relaciona o ISA/Município à população total dos municípios:

ISA/Município $=17,17+8,27 \ln \left(\right.$ Pop $\left._{\text {total }}\right)$
A Figura 5 mostra o ajuste da Equação (4) aos dados obtidos. Vale destacar que foram excluídos da correlação os municípios com condições atípicas de abastecimento de água via Sistema Integrado de Saneamento Rural - SISAR, isto é, Altaneira, que possui índice de atendimento com rede de distribuição de água tratada na zona rural superior a $85 \%$, e Caririaçu e Jardim, que possuem índice inferior a $5 \%$. 


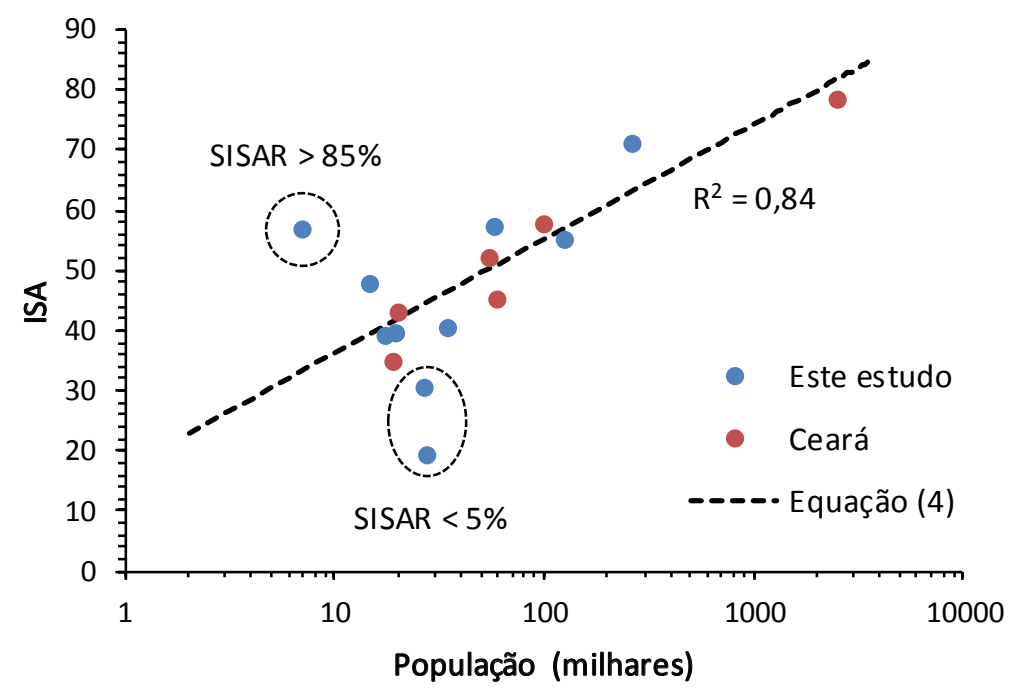

Figura 5 - Correlação entre o Índice de Salubridade Ambiental (ISA/Município) e a população de municípios do Ceará. Os círculos representam os municípios com condições atípicas de abastecimento de água via Sistema Integrado de Saneamento

Rural - SISAR, isto é, Altaneira, que possui índice de atendimento com rede de distribuição de água tratada na zona rural superior a 85\%, e Caririaçu e Jardim, que possuem índice inferior a 5\%. Esses três municípios foram excluídos da correlação.

Cabe salientar que no presente estudo também foram analisadas correlações com o índice de desenvolvimento humano (IDH), Produto Interno Bruto (PIB), entre outros. A melhor correlação obtida, porém, foi em função da população dos municípios. Além disso, a população é a variável de mais fácil obtenção. Portanto, decidiu-se por adotar a população como variável de referência.
Como exemplo de aplicação da Equação (4), a Figura 6 mostra uma extrapolação da correlação obtida para os 184 municípios do Estado do Ceará. Os resultados sugerem que $84 \%$ dos municípios estejam na classe de baixa salubridade, $15 \%$ na classe de média salubridade, e apenas $1 \%$, o que corresponde à capital do Estado (Fortaleza), classificado como de salubridade aceitável.

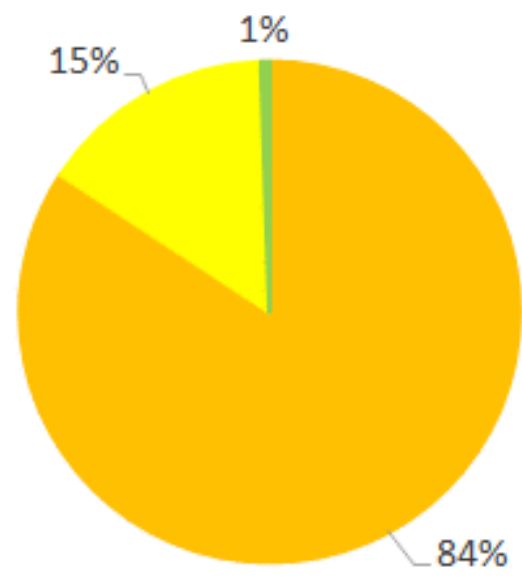

\section{Baixa salubridade Média salubridade Salubridade aceitável}

Figura 6 - Aplicação da correlação obtida (Equação 4) para avaliação do Índice de Salubridade Ambiental (ISA/ Município) nos 184 municípios do Ceará. 


\section{CONCLUSÕES}

A pesquisa apresentou uma metodologia para avaliação do Índice de Salubridade Ambiental (ISA) em função do nível de atendimento dos serviços de saneamento, conforme detalhado nos Planos Municipais de Saneamento Básico (PMSB), englobando tanto as zonas urbanas como as zonas rurais. Foram avaliados dez municípios da região centro-sul do Estado do Ceará, com populações variando entre 7 mil e 265 mil habitantes. Os principais resultados do estudo são apresentados a seguir:

- Em todos os municípios avaliados, as zonas rurais apresentaram níveis de salubridade inferiores aos das zonas urbanas;

- Os municípios foram em geral classificados como $10 \%$ insalubres, $50 \%$ de baixa salubridade e $40 \%$ de média salubridade;

- Verificou-se que quanto mais populoso for o município, melhor a salubridade ambiental em função de maiores investimentos alocados na área de saneamento básico;

- Verificou-se que a existência de Sistema Integrado de Saneamento Rural (SISAR) foi um fator determinante para a classificação da salubridade ambiental dos municípios de menor porte;

- Observou-se que somente a partir de um horizonte de planejamento de médio prazo (20 anos) os municípios mais populosos poderão atingir a condição de salubres;

- Ajustou-se uma correlação geral capaz de descrever o ISA/Município em função da população dos municípios;

- A extrapolação dessa correlação para todo o Estado do Ceará sugere que cerca de $84 \%$ dos municípios estejam com baixa salubridade.
Em síntese, verificou-se a necessidade de realização de investimentos massivos e de forma continuada no setor de saneamento básico, de modo a buscar a universalização do setor no Estado do Ceará e a atingir pelo menos a condição de salubridade aceitável dentro do horizonte previsto nos PMSB (30 anos).

Dispondo de dados confiáveis, a metodologia proposta também poderá ser testada e, se necessário, adaptada para avaliar o comportamento da salubridade ambiental em outros municípios brasileiros ou mesmo em nível de estados da federação.

\section{REFERÊNCIAS}

ALTANEIRA. Plano de Saneamento Básico de Altaneira. Fundação Nacional de Saúde - FUNASA - 2012.

BARBALHA. Plano de Saneamento Básico de Barbalha. Fundação Nacional de Saúde - FUNASA - 2012.

BATISTA, M. E. M.; SILVA, T. C. O modelo ISA/JP - Indicador de Performance para Diagnóstico do Saneamento Ambiental Urbano. Engenharia Sanitária e Ambiental, v. 2, p. 55-64, 2006.

BRASIL. DECRETO No 7.217 de 21 de junho de 2010. Regulamenta a Lei $n^{\circ} 11.4445$, de 5 de janeiro de 2007, que estabelece diretrizes nacionais para o saneamento básico, e dá outras providências.

BRASIL. LEI No 11.445 de 5 de janeiro de 2007. Estabelece diretrizes nacionais para o saneamento básico; altera as Leis nos 6.766, de 19 de dezembro de 1979, 8.036, de 11 de maio de 1990, 8.666, de 21 de junho de 1993, 8.987, de 13 de fevereiro de 1995; revoga a Lei no 6.528 , de 11 de maio de 1978; e dá outras providências.

BUCKLEY, C. F. O.; DALTRO FILHO, J. Adaptação do Indicador de Salubridade Ambiental - ISA para análise de empreendimentos do Programa de Arrendamento Residencial - PAR em Aracaju/SE. Revista DAE, 189, 16-25, 2012.

CARIRIAÇU. Plano de Saneamento Básico de Caririaçu. Fundação Nacional de Saúde - FUNASA - 2012.

CONESAN. Indicador de Salubridade Ambiental, ISA. Manual de Orientação Técnica. Companhia de Saneamento Básico do Estado de São Paulo (Sabesp), 1999.

COSTA, R. V. F. Desenvolvimento do Índice de Salubridade Ambiental (ISA) para comunidades rurais e sua aplicação e análise nas comunidades de Ouro Branco/MG. Dissertação (Mestrado em Engenharia Ambiental) - Universidade Federal de Ouro Preto, 2010. 
CRATO. Plano de Saneamento Básico de Crato. Fundação Nacional de Saúde - FUNASA - 2012.

CUNHA, T. B. Análise integrada da salubridade ambiental e condições de moradia: Aplicação no município de Itaguaçu na Bahia. Dissertação (Mestrado em Engenharia Urbana e Ambiental) - Universidade Federal da Paraíba, 2012.

DIAS, M. C.; BORJA, P. C.; MORAES, L. R. C. Índice de Salubridade Ambiental em áreas de ocupação espontâneas: um estudo em Salvador - Bahia. Engenharia Sanitária e Ambiental, v. 9, p. 8292, 2004.

FARIAS BRITO. Plano de Saneamento Básico de Farias Brito. Fundação Nacional de Saúde - FUNASA - 2012.

GALVÃO JUNIOR, A. C. Planos Municipais de Saneamento Básico. Revista DAE, 193, 6-15, 2014.

HELLER, L. Relação entre saúde e saneamento na perspectiva do desenvolvimento. Ciência \& Saúde Coletiva, 3(2): 73-84, 1997.

JARDIM. Plano de Saneamento Básico de Jardim. Fundação Nacional de Saúde - FUNASA - 2012.

JUAZEIRO DO NORTE. Plano de Saneamento Básico de Juazeiro do Norte. Fundação Nacional de Saúde - FUNASA - 2012.

LIMA NETO, I. E. Planejamento no Setor de Saneamento Básico Considerando o Retorno da Sociedade. Revista DAE, 46-52, 2011.

LIMA NETO, I. E. Proposta metodológica para análise de viabilidade econômico-financeira da universalização e prestação dos serviços de saneamento básico. Revista DAE, 193, 56-65, 2013.

LIMA NETO, I. E.; DOS SANTOS, A. B. Planos de Saneamento Básico. In: Gestão de Saneamento Básico. PHILIPPI JR., A.; GALVÃO JÚNIOR, A. C., Manole, 57-79, 2011.

MISSÃO VELHA. Plano de Saneamento Básico de Missão Velha. Fundação Nacional de Saúde - FUNASA - 2012.
NOVA OLINDA. Plano de Saneamento Básico de Nova Olinda. Fundação Nacional de Saúde - FUNASA - 2012.

PEREIRA, T. S. T., HELLER, L. Planos municipais de saneamento básico: avaliação de 18 casos brasileiros. Engenharia Sanitária e Ambiental, v. 20(3), p. 395-404, 2015.

PHILIPPI JR., A. Saneamento, saúde e ambiente. Manole, 842 p, 2005.

PHILIPPI JR., A., MALHEIROS, T. F.; AQUIAR, A. O. Indicadores de desenvolvimento sustentável. In: Saneamento, saúde e ambiente. PHILIPPI JR., A., Manole, 761-808, 2005.

RIBEIRO, M. A. P. Estudo comparativo entre uma ocupação espontânea urbanizada e outra não urbanizada na cidade de Fortaleza com base no modelo ISA/F (Indicador de Salubridade Ambiental em Favelas). Dissertação (Mestrado em Engenharia Civil) - Centro de Tecnologia, Departamento de Engenharia Hidráulica e Ambiental, Universidade Federal do Ceará, Fortaleza, 2007.

SANTANA DO CARIRI. Plano de Saneamento Básico de Santana do Cariri. Fundação Nacional de Saúde - FUNASA - 2012.

SRH-CE, Secretaria Estadual de Recursos Hídricos do Ceará (2016). http://atlas.srh.ce.gov.br. Acesso em 11/08/2016.

TEIXEIRA, J. C.; GOMES, M. H. R.; SOUZA, J. A. Análise da Associação entre Saneamento e Saúde nos Estados Brasileiros - estudo comparativo entre 2001 e 2006. Engenharia Sanitária e Ambiental, v. 16, p. 197-204, 2011.

VALVASSORI, M. L.; ALEXANDRE, N. Z. Aplicação do Indicador de Salubridade Ambiental (ISA) para áreas urbanas. Revista Brasileira de Ciências Ambientais, 25, 1-19, 2012.

VARGAS, C. V. Universalização dos Serviços de Saneamento Básico. In: Gestão de Saneamento Básico. PHILIPPI JR., A.; GALVÃO JÚNIOR, A. C., Manole, 721-750, 2011. 